Review Article

\title{
Metformin Use Is Associated with Reduced Incidence and Improved Survival of Endometrial Cancer: A Meta-Analysis
}

\author{
Yun-Liang Tang, ${ }^{1}$ Ling-Yan Zhu, ${ }^{1}$ Yu Li, ${ }^{2}$ Jiao Yu, ${ }^{1}$ Jiao Wang, ${ }^{1}$ Xiang-Xia Zeng, \\ Kai-Xiang $\mathrm{Hu}^{1}{ }^{1}$ Jian-Ying Liu, ${ }^{1}$ and Ji-Xiong $\mathrm{Xu}^{1}$ \\ ${ }^{1}$ Department of Endocrinology and Metabolism, First Affiliated Hospital of Nanchang University, Nanchang, Jiangxi 330006, China \\ ${ }^{2}$ Department of Pharmacy, Pingxiang Maternal and Child Health Care Hospital, Pingxiang, Jiangxi 337000, China
}

Correspondence should be addressed to Ji-Xiong Xu; xujixiong@163.com

Received 2 November 2016; Revised 31 December 2016; Accepted 24 January 2017; Published 20 March 2017

Academic Editor: Takashi Yazawa

Copyright (C) 2017 Yun-Liang Tang et al. This is an open access article distributed under the Creative Commons Attribution License, which permits unrestricted use, distribution, and reproduction in any medium, provided the original work is properly cited.

Studies have suggested that metformin can potentially decrease the incidence of cancer and improve survival outcomes. However, the association between metformin use and the incidence and survival of endometrial cancer (EC) remains controversial. So, a metaanalysis was performed. An electronic search was conducted using PubMed, EMBASE, and Web of Science. The outcome measures were relative risks (RRs) or hazard ratios (HRs) with 95\% confidence intervals (CIs) comparing the EC incidence and survival in patients treated with and without metformin. Eleven studies involving 766,926 participants were included in this study. In the pooled analysis of five studies which evaluated the association of metformin use with the incidence of EC, we found that metformin use was associated with a $13 \%$ reduction in EC risk among patients with diabetes ( $\mathrm{RR}=0.87,95 \% \mathrm{CI}: 0.80-0.95 ; p=0.006)$. In the pooled analysis of six retrospective cohort studies evaluating the effect of metformin on the survival of EC patients, we found that, relative to nonuse, metformin use significantly improved the survival of EC patients ( $\mathrm{HR}=0.63,95 \% \mathrm{CI}: 0.45-0.87 ; p=0.006$ ). This study showed that metformin use was significantly associated with a decreased incidence of EC in diabetes and a favorable survival outcome of EC patients.

\section{Introduction}

Endometrial cancer (EC) is the most commonly occurring gynecologic malignant tumor of the female reproductive system, and its incidence is increasing worldwide $[1,2]$. Both diabetes and obesity are risk factors which promote the development and progression of EC [3]. Primary surgical treatment is the mainstay of therapy, including total hysterectomy and bilateral salpingooophorectomy [4]. Unfortunately, many patients diagnosed with local or advanced EC will still suffer from recurrence or die of this disease, although some of them had been cured. Thus, many efforts are needed to reduce the incidence of EC as well as research to identify novel therapeutic targets with the aim of improving the disease survival.

Metformin, an oral biguanide, one of the most commonly prescribed antidiabetic medications [5], may have antineoplastic properties. Recent epidemiological studies have reported potential beneficial effects of metformin on gynecological cancers. Metformin use significantly decreased the risk or improved some survival outcomes among patients with cervical, breast, and ovarian cancer [6-8]. Moreover, several meta-analyses have shown that metformin use decreased the incidence and improved the survival of a wide range of malignant tumors, such as liver cancer, lung cancer, prostate cancer, and colorectal cancer [9-15]. Although the exact mechanism is still not fully understood, studies in vivo and vitro have shown that metformin likely exerts its antitumorigenic effects directly or through other downstream targets to inhibit the growth and proliferation of tumor cells. Direct mechanisms may be the activation of 5-AMP-activated protein kinase (AMPK), which results in the inactivation of mammalian target of rapamycin (mTOR) [16]. Indirect mechanisms may be the inhibition of liver gluconeogenesis, resulting in a decrease in insulin levels and hyperglycemia [17]. 
Thus far, several clinical researches have investigated the effect of metformin on the incidence of EC in diabetic patients. However, it is still uncertain whether the use of metformin could decrease the incidence of EC owing to the contradictory results of these studies. Tseng reported that the use of metformin in women with diabetes was associated with an overall significantly lower risk of EC [18]. However, Franchi et al.s study indicated that metformin did not meaningfully affect the risk of EC [19]. Moreover, studies have also investigated the association between metformin use and survival of EC patients. However, whether metformin use could generate better clinical outcomes in EC patients also remains unclear. Ko et al. reported that the nonmetformin users had 2.3-fold increased risk of death when compared with the metformin users after adjusting for age, stage, grade, histology, and adjuvant treatment [20]. Conversely, Al Hilli et al. reported that overall survival was similar between the metformin users and nonusers of EC patients after adjusting for confounding covariates [21]. Based on these studies, the association between metformin use and the incidence and survival of EC is still uncertain; thus, a meta-analysis is needed to confirm the effects of metformin.

\section{Materials and Methods}

2.1. Literature Search. A comprehensive literature search was performed to identify all potentially relevant articles using the PubMed, EMBASE, and Web of Science database from their inception to 20 October 2016. The search was restricted to the articles published in English. We developed a search strategy using the following terms: "metformin" or "biguanide" and "endometrial cancer" or "endometrial carcinoma". Additionally, we screened bibliographies of the selected original studies and review articles to identify any other relevant studies that were not captured through the initial database searches.

2.2. Eligibility Criteria. Studies were included if they met the following criteria: (a) randomized controlled clinical trials, case-control studies, nested case-control studies, and cohort studies; (b) studies evaluating the association between metformin use and incidence of EC in diabetes or those evaluating the effect of metformin on survival of EC patients; (c) studies reporting relative risks (RRs) or hazard ratios (HRs) with corresponding 95\% confidence intervals (CIs) or providing sufficient data to calculate these values. If more than one paper was based on the same study, we included the one which provided the most abundant information or the one containing the largest number of cases. Studies were excluded if they were publications from letters, editorials, reviews, cell line studies, animal studies, and studies without controls.

2.3. Data Extraction Quality Assessment. Two authors performed the data extraction independently. For the eligibility studies, the collected information included first author, year of publication, region, study design, data source, sample size, time period, RR (95\% CI) or HR (95\% CI), and variables controlled for matching or used in multivariable models. If several estimates were reported in the same article, the most fully adjusted estimate was selected. Disagreements were resolved by discussion between two reviewers. When required, disagreements were resolved by consultation with a third reviewer $(\mathrm{Xu})$.

To assess the quality of included studies, the NewcastleOttawa Scale (NOS) was applied in this meta-analysis [29]. We evaluated the included studies based on selection of participants, comparability of participants, and ascertainment of outcomes and then scored the methodological quality. Quality assessment was independently conducted by two authors, with a third party $(\mathrm{Xu})$ assessment when necessary.

2.4. Statistical Analyses. To assess the association between metformin use and the incidence and survival of EC, summary RRs and HRs with their corresponding 95\% CIs were calculated through meta-analysis. Heterogeneity analysis was performed by Cochran $Q$ statistic and $I^{2}$ statistic. Statistical significance for heterogeneity was considered if $p<0.05$ or $I^{2}>50 \%$. The fixed-effects model was applied when $p>0.05$ and $I^{2}<50 \%$, while the random-effects model was chosen when $p<0.05$ or $I^{2}>50 \%$. To identify the sources of heterogeneity, we performed subgroup analyses. Additionally, we conducted sensitivity analyses by removing one study each time and recalculating pooled effects. Potential publications bias (considered present if $p \leq 0.1$ ) was assessed by conducting statistical tests for funnel plot asymmetry as well as Egger's test and Begg's test. All statistical tests were conducted using Stata software (Version 12; StataCorp, College Station, TX, USA).

\section{Results}

3.1. Study Characteristics. The participant flow diagram for the study inclusion in the metaanalysis is shown in Figure 1. Finally, 11 relevant studies were retrieved, comprising a total of approximately 766,926 participants. Five studies referred to the incidence of EC with a total of 764,810 participants $[18,19,22-24]$. Six studies investigated the survival of EC with 2,116 patients [20, 21, 25-28]. Information on region, data source, time period, sample size, and adjustment variables is presented in Tables 1 and 2. Nine studies were designed as retrospective cohort studies, one was nested case-control study, and one was case-control study. Five studies were based in Poland, Austria, Taiwan, UK, and Italy, respectively. Six studies were based in the USA. Of the included studies, adjusted multivariate analyses for the effect of metformin were performed in 9 studies, and unadjusted univariate analyses were performed in 2 studies. The NOS score of the selected studies ranged from 6 to 8 stars on the scale, which suggested moderate to high quality.

\subsection{Quantitative Synthesis}

3.2.1. Metformin Use and Incidence of Endometrial Cancer. Based on the combined results of the five studies, compared 


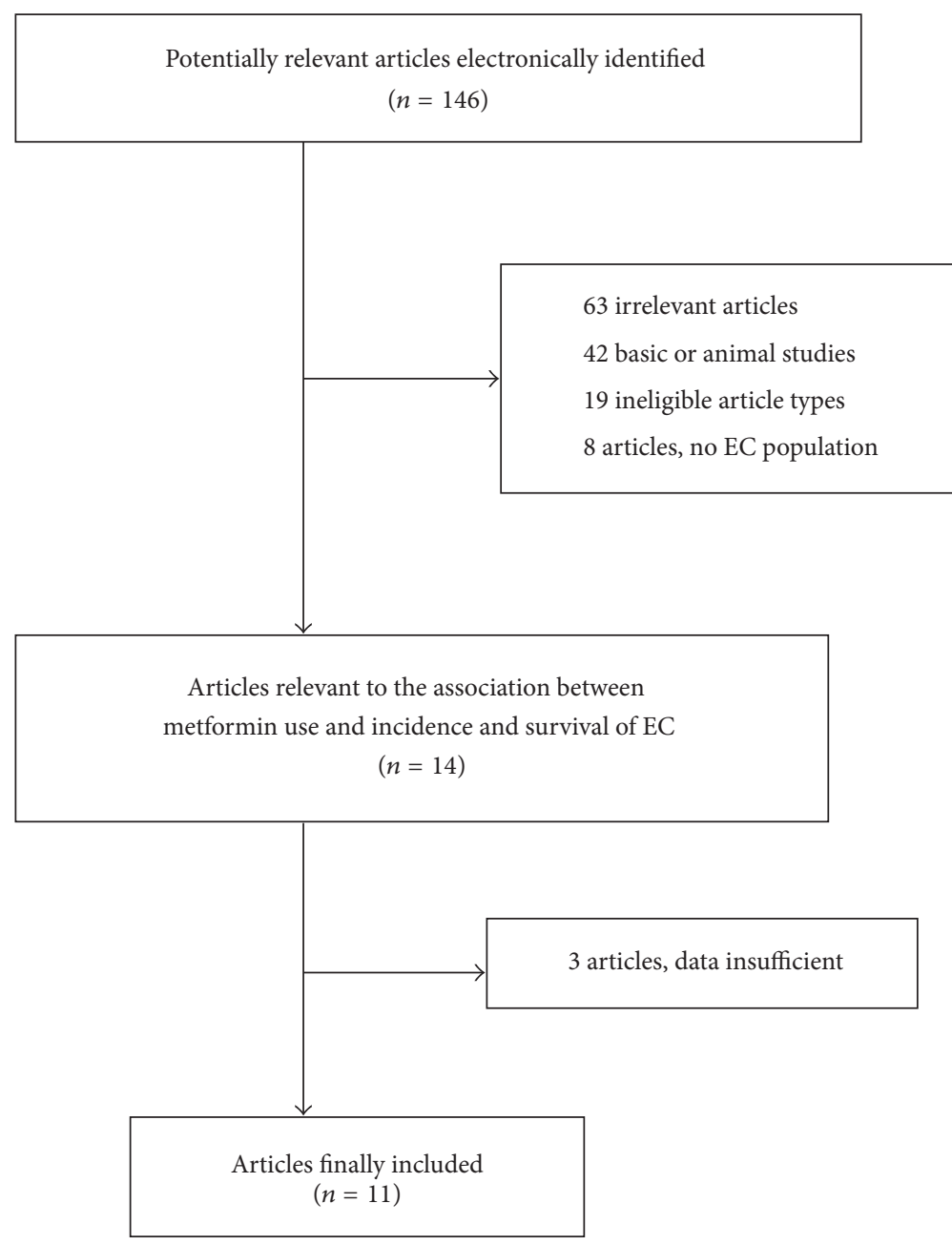

Figure 1: Flow diagram of study selection.

with the reference groups, metformin use was significantly associated with a decreased incidence of $\mathrm{EC}$ in diabetes $(\mathrm{RR}=$ 0.87, 95\% CI: 0.80-0.95; $p=0.006$; Figure 2). Moreover, there was no obvious between-study heterogeneity in this metaanalysis of five studies in total $\left(I^{2}=0.0 \%\right)$. Stratification according to study design showed that metformin use was significantly associated with a decreased incidence of EC for retrospective studies $(\mathrm{RR}=0.85,95 \% \mathrm{CI}: 0.78-0.93 ; p=$ $\left.0.001 ; I^{2}=0.0 \%\right)$, but not for case-control studies $(\mathrm{RR}=0.88$, 95\% CI: $0.58-1.32, p=0.542)$ or nested case-control studies $(\mathrm{RR}=1.07,95 \% \mathrm{CI}: 0.82-1.41, p=0.625)$.

3.2.2. Metformin Use and Survival of Endometrial Cancer. Compared with no metformin use, metformin use was associated with survival improvement of EC patients (HR $=0.63$, 95\% CI: 0.45-0.87; $p=0.006$; Figure 3). Figure 3 also shows the HRs (95\% CI) for each individual study comparing the group of metformin use with the reference group (no metformin use). There was obvious between-study heterogeneity in this meta-analysis of six studies in total $\left(I^{2}=52.0 \%\right)$. In the stratified analyses by adjustment, pooled analysis of four studies with adjustment of variables showed the same effect $(\mathrm{HR}=0.49,95 \% \mathrm{CI}: 0.36-0.46 ; p=$ $0.000)$ with low heterogeneity $\left(I^{2}=0.0 \%\right)$. However, two studies without adjusting variables showed that metformin use was not associated with the overall survival of patients with $\mathrm{EC}\left(\mathrm{HR}=0.92,95 \% \mathrm{CI}: 0.70-1.19 ; p=0.512 ; I^{2}=\right.$ $0.0 \%)$. Stratified analyses by reference group showed that the beneficial effect of metformin use was stable ( $\mathrm{HR}=0.47,95 \%$ CI: $0.33-0.67 ; p=0.000 ; I^{2}=0.0 \%$ ), when the controlled nonmetformin users were restricted to EC patients with diabetes. However, when compared with nonmetformin users with and without diabetes, the beneficial effect on survival lost significance ( $\mathrm{HR}=0.84,95 \% \mathrm{CI}$ : $0.66-1.07 ; p=0.149$; $\left.I^{2}=27.2 \%\right)$.

3.2.3. Sensitivity Analysis. Sensitivity analyses were conducted to verify the effect of each study on the overall estimate by omitting one study at a time and calculating the combined results for the remaining studies. Finally, we found that no individual study significantly affected the pooled RR and HR.

3.2.4. Publication Bias. To assess the possibility of publication bias among the studies, funnel plots were generated 


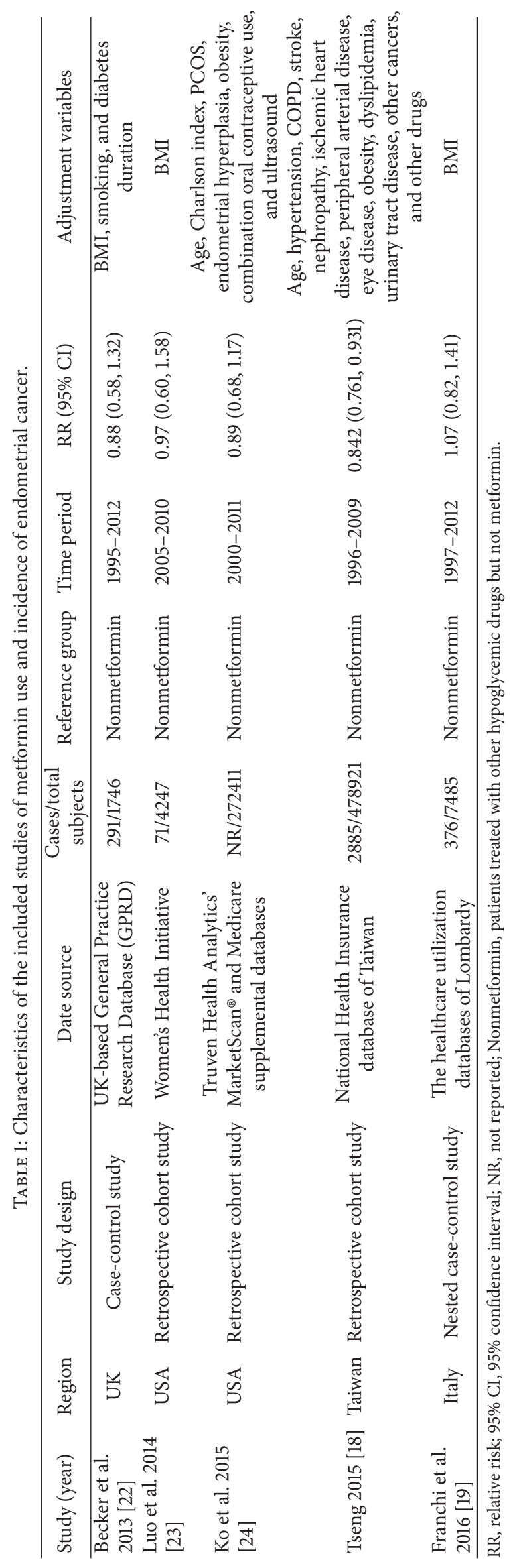




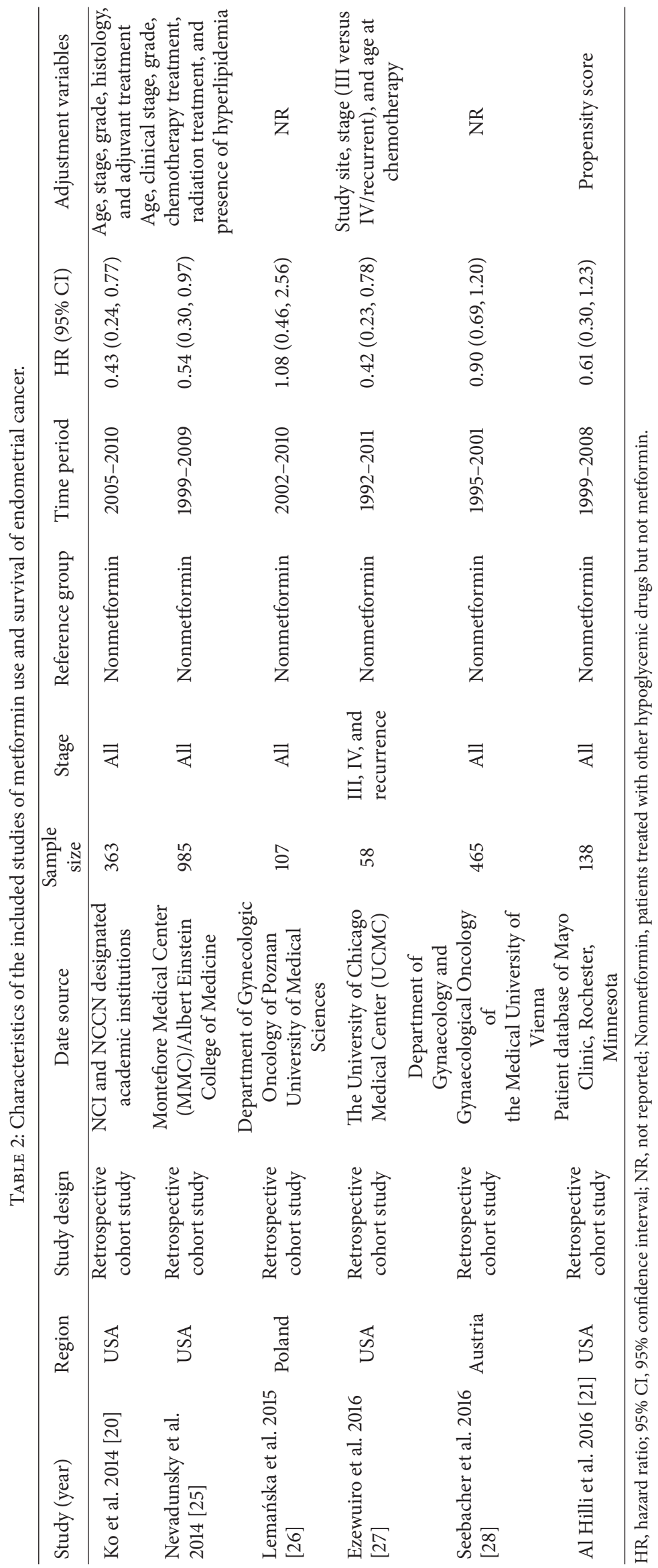




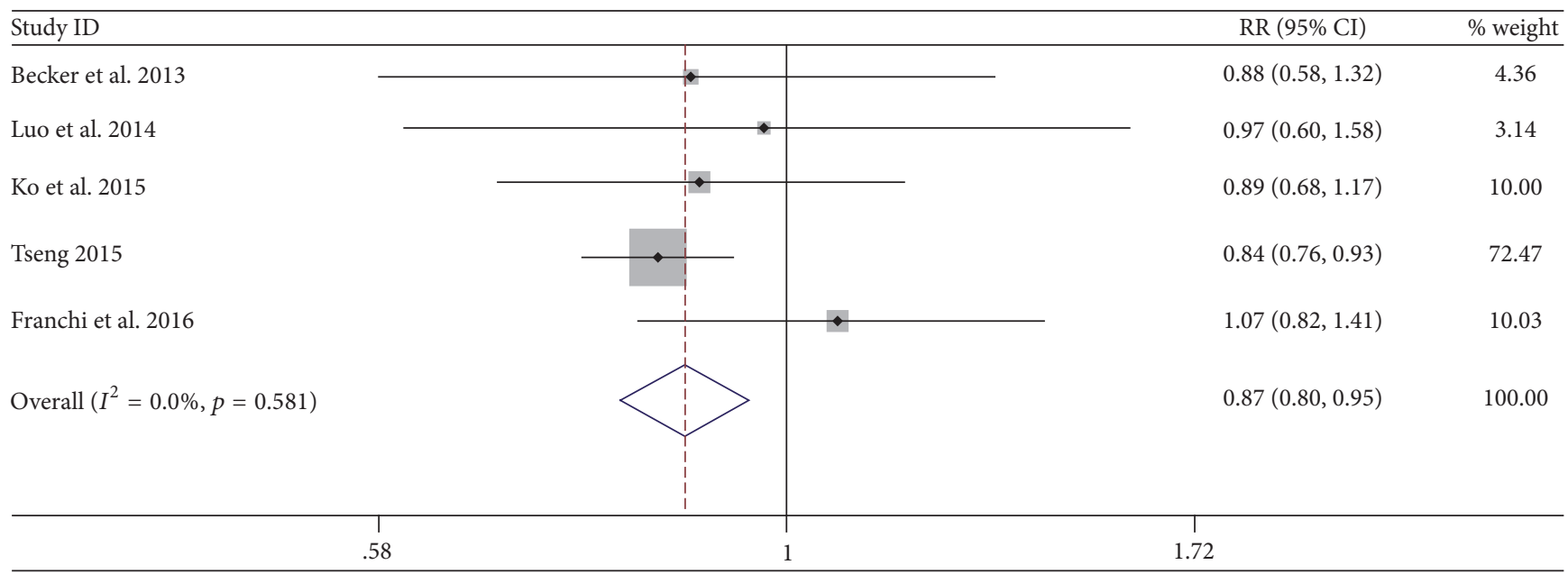

FIGURE 2: Forest plot of the association between metformin use and incidence of endometrial cancer.

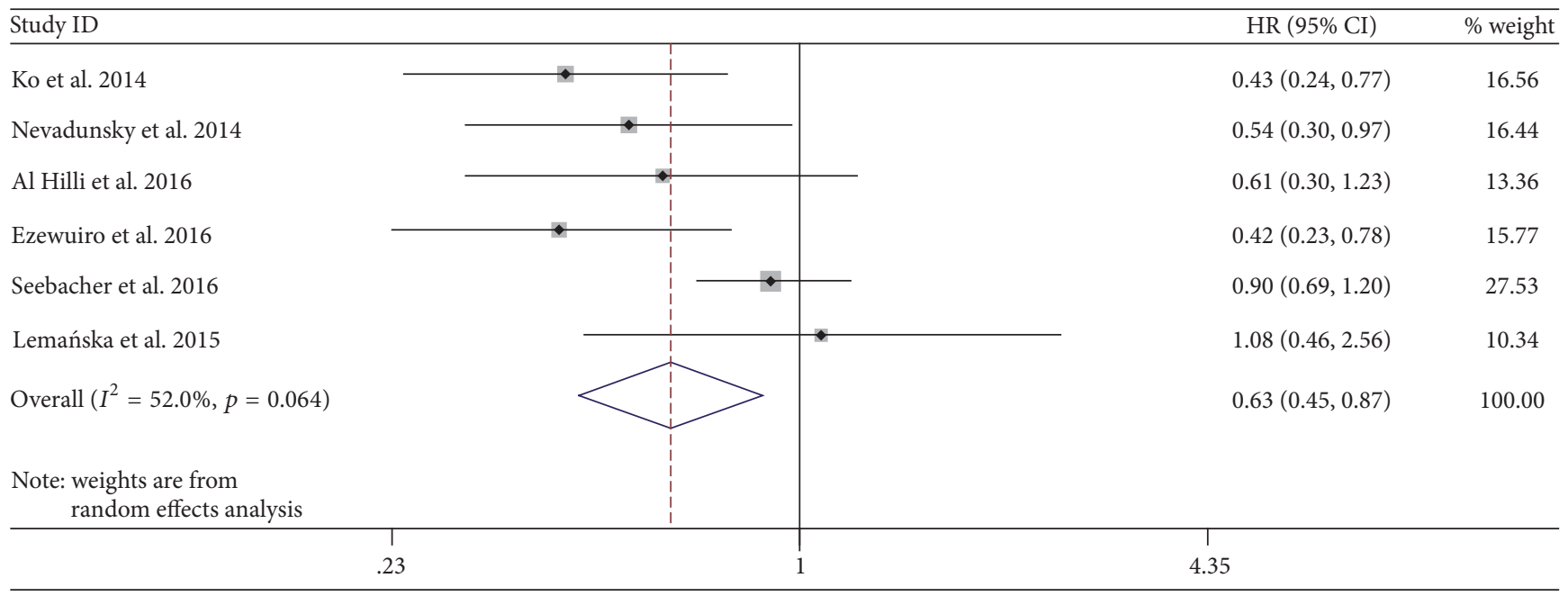

FIGURE 3: Forest plot of the association between metformin use and survival of endometrial cancer.

(Figures 4 and 5). Finally, no evident asymmetry of the funnel plot was detected, indicating that there was no obvious publication bias in our study, which was also supported by Egger's test $(p=0.231$ and $p=0.220$ for incidence and survival, resp.) and Begg's test ( $p=0.707$ and $p=0.806$ for incidence and survival, resp.).

\section{Discussion}

In this study, we analyzed the association of metformin use with the incidence and survival of EC using a metaanalysis to obtain a powerful conclusion. To the best of our knowledge, this is the first meta-analysis providing comprehensive insights into the effects of metformin use on the incidence and survival of EC. In the pooled analysis of five studies which evaluated the association of metformin use with the incidence of EC, we found that metformin use was associated with a $13 \%$ reduction in EC risk among patients with diabetes $(\mathrm{RR}=0.87,95 \% \mathrm{CI}: 0.80-0.95 ; p=0.006)$. In the pooled analysis of six retrospective cohort studies which investigated the effect of metformin use on the survival of EC patients, we found that, relative to nonuse, metformin use significantly improved the survival of EC patients $(\mathrm{HR}=0.63$, 95\% CI: 0.45-0.87; $p=0.006$ ).

Metformin, a biguanide, has become the most widely used antihyperglycemic drug. This drug is characterized by a broad spectrum of pleiotropic effects and good tolerability by patients. It may inhibit the growth and proliferation of gynecological cancer cells, such as those in breast cancer [30], ovary cancer [31], and cervical cancer [32]. In the past decade, many epidemiological studies showed the association between metformin use and the reduced risk and improved survival of patients with several types of cancers, including gynecological cancers. The beneficial effects of metformin use on EC may depend on common anticancer mechanisms present in other gynecological cancers [33] and even all tumors [34], but the exact molecular mechanism has not yet been fully elucidated. 


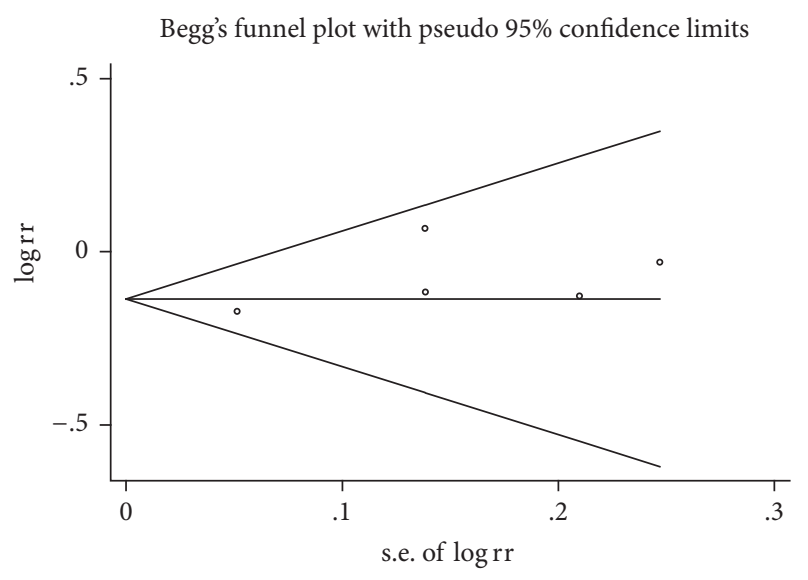

FIGURE 4: Begg's funnel plots for publication bias test on the association of metformin use with the incidence of endometrial cancer ( $p=0.231$ for Egger's test and $p=0.707$ for Begg's test).

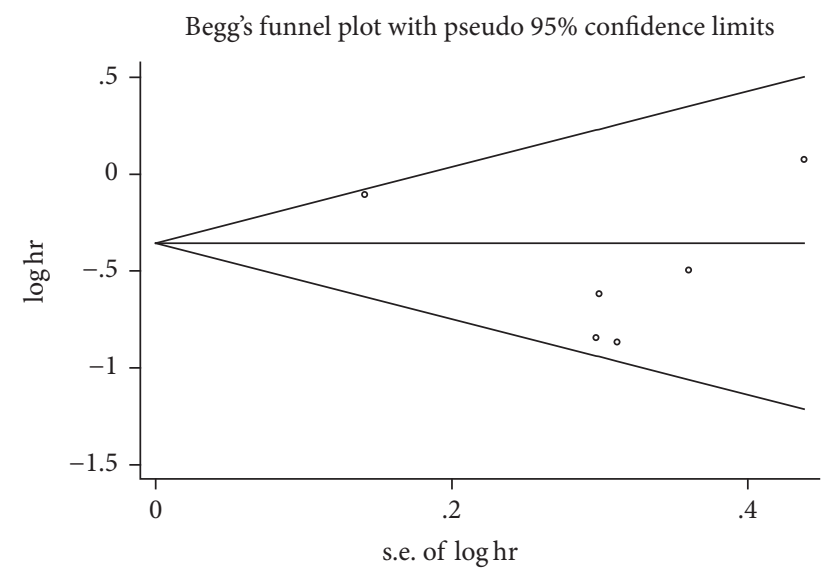

FIGURE 5: Begg's funnel plots for publication bias test on the association of metformin use with the survival of endometrial cancer ( $p=0.220$ for Egger's test and $p=0.806$ for Begg's test).

The most frequently proposed hypotheses are the indirect effects of reducing levels of insulin and even insulin-like growth factor 1 (IGF-1), which is closely related to insulin signaling and exerts direct effects on the activation of cellular pathways of tumor cells. Metformin use reduced the blood glucose levels by the inhibition of gluconeogenesis in the liver [35], resulting in lowered circulating insulin levels. Epidemiological studies suggested that a high insulin level was associated with increased occurrence and mortality of cancers [36-38]. IGF-1 also plays a role in the occurrence and development of tumors [39], and the level of IGF-1 is significantly higher in EC compared with that in the normal endometrium [40]. Insulin/IGF-1 signaling is initiated by its binding to transmembrane receptors, which results in activation of tyrosine kinase activity. As with the activation of tyrosine kinase activity, adaptor proteins, such as the P85, are subsequently activated. P85 is the regulatory subunit of phosphatidylinositol 3-kinase (PI3-K) [41]. Insulin/IGF1/PI3-K pathway plays a role in the carcinogenesis via mTOR, which is a key effector of PI3-K. In up to $80 \%$ of human cancers, mTOR is aberrantly activated [42]. Moreover, metformin use can also exert its direct antitumorigenic effects by activating AMPK, which participates in cellular proliferation and metabolism, including the inhibition of mTOR pathway [43]. mTOR plays a key role in cell growth and proliferation. Additionally, it participates in the formation of two protein complexes: mTOR complexes 1 and 2 (mTORC1/2). mTORC1 signaling is switched on by several oncogenic signaling pathways and is hyperactive in the majority of cancers [44].

The current meta-analysis has several strengths. First, the major strength of this study is that we comprehensively assessed the effects of metformin use on EC, using incidence and survival as the primary outcomes. Second, no obvious heterogeneity was detected in the evaluation of incidence of EC $\left(I^{2}=0.0 \%\right)$. Moreover, in the subgroup analyses of survival, the values of $I^{2}$ were all less than $25 \%$. Third, the sensitivity analysis did not show that a single study influenced the pooled results, and no publication bias was detected. Given these characteristics, this meta-analysis can be considered the most comprehensive study of metformin effects on EC, thus far.

However, several limitations in our study should be acknowledged. First, two of the eligible studies included in this meta-analysis did not report adjustments and the adjustments of the other nine studies were either inconsistent or incomplete. Many other confounders, such as cumulative dose, use of concomitant medications, and time-related bias, were not controlled. These aspects would be important to provide a more in-depth understanding of the nature of metformin use [45]. Second, the population of this study was based on Western and Asian populations. The lack of data from South America and Africa could possibly limit the generalizability of our conclusions. Therefore, it is necessary to verify the results in these areas. Moreover, this study was restricted to publications in English, which might also introduce publication bias. Finally, even though we found an obvious association between metformin use and the survival of patients with EC, current studies are unable to provide a conclusive result, because the sample size included in our meta-analysis is not sufficiently large. Thus, more prospective studies with large sample sizes are warranted.

\section{Conclusions}

In summary, our meta-analysis of observational studies demonstrated that metformin use reduces the incidence of EC among patients with diabetes and improves the overall survival of patients with EC. Our study results suggest that metformin may be a potential alternative treatment for patients with diabetes at high risk of EC and patients with EC and concomitant diabetes. However, accounting for the limitations of observational studies and the other limitations mentioned above, causality cannot be established yet. Further studies with large sample sizes, especially blind, randomized controlled clinical trials, are needed to confirm these results. 


\section{Conflicts of Interest}

The authors declare that no conflicts of interest exist.

\section{Authors' Contributions}

Yun-Liang Tang and Ling-Yan Zhu contributed equally to this work.

\section{Acknowledgments}

The study was supported by grants from the National Natural Science Funds of China (nos. 81460018, 81560154, and 81160105), Jiangxi Provincial Science Technology Foundation of China (no. 20151BBG70073), and Jiangxi Provincial Department of Education Scientific Research Funds of China (nos. GJJ13145 and GJJ13178).

\section{References}

[1] R. L. Siegel, K. D. Miller, and A. Jemal, "Cancer statistics, 2015," CA Cancer Journal for Clinicians, vol. 65, no. 1, pp. 5-29, 2015.

[2] A. Jemal, F. Bray, M. M. Center, J. Ferlay, E. Ward, and D. Forman, "Global cancer statistics," CA: A Cancer Journal for Clinicians, vol. 61, no. 2, pp. 69-90, 2011.

[3] V. M. Chia, P. A. Newcomb, A. Trentham-Dietz, and J. M. Hampton, "Obesity, diabetes, and other factors in relation to survival after endometrial cancer diagnosis," International Journal of Gynecological Cancer, vol. 17, no. 2, pp. 441-446, 2007.

[4] J. D. Wright, N. I. B. Medel, J. Sehouli, K. Fujiwara, and T. J. Herzog, "Contemporary management of endometrial cancer," The Lancet, vol. 379, no. 9823, pp. 1352-1360, 2012.

[5] D. M. Nathan, J. B. Buse, M. B. Davidson et al., "Management of hyperglycaemia in type 2 diabetes: a consensus algorithm for the initiation and adjustment of therapy. A consensus statement from the American Diabetes Association and the European Association for the Study of Diabetes," Diabetologia, vol. 49, no. 8, pp. 1711-1721, 2006.

[6] J. Hanprasertpong, I. Jiamset, A. Geater, T. Peerawong, W. Hemman, and S. Kornsilp, "The effect of metformin on oncological outcomes in patients with cervical cancer with Type 2 diabetes mellitus," International Journal of Gynecological Cancer, vol. 27, no. 1, pp. 131-137, 2017.

[7] H. Xu, M. C. Aldrich, Q. Chen et al., "Validating drug repurposing signals using electronic health records: a case study of metformin associated with reduced cancer mortality," Journal of the American Medical Informatics Association, vol. 22, no. 1, pp. 179-191, 2015.

[8] S. Kumar, A. Meuter, P. Thapa et al., "Metformin intake is associated with better survival in ovarian cancer: a case-control study," Cancer, vol. 119, no. 3, pp. 555-562, 2013.

[9] S. J. Ma, Y. X. Zheng, P. C. Zhou, Y. Xiao, and H. Tan, "Metformin use improves survival of diabetic liver cancer patients: systematic review and meta-analysis," Oncotarget, vol. 7, no. 40, pp. 66202-66211, 2016.

[10] G. Wan, X. Yu, P. Chen et al., "Metformin therapy associated with survival benefit in lung cancer patients with diabetes," Oncotarget, vol. 7, no. 23, pp. 35437-35445, 2016.

[11] K. H. Stopsack, D. R. Ziehr, J. R. Rider, and E. L. Giovannucci, "Metformin and prostate cancer mortality: a meta-analysis," Cancer Causes and Control, vol. 27, no. 1, pp. 105-113, 2016.
[12] X.-K. He, T.-T. Su, J.-M. Si, and L.-M. Sun, "Metformin is associated with slightly reduced risk of colorectal cancer and moderate survival benefits in diabetes mellitus: a metaanalysis," Medicine, vol. 95, no. 7, article e2749, 2016.

[13] Z.-J. Zhang, Z.-J. Zheng, R. Shi, Q. Su, Q. Jiang, and K. E. Kip, "Metformin for liver cancer prevention in patients with type 2 diabetes: a systematic review and meta-analysis," Journal of Clinical Endocrinology and Metabolism, vol. 97, no. 7, pp. 23472353, 2012.

[14] N. Zhu, Y. Zhang, Y. I. Gong, J. He, and X. Chen, "Metformin and lung cancer risk of patients with type 2 diabetes mellitus: a meta analysis," Biomedical Reports, vol. 3, no. 2, pp. 235-241, 2015.

[15] H. Yu, L. Yin, X. Jiang et al., "Effect of metformin on cancer risk and treatment outcome of prostate cancer: a meta-analysis of epidemiological observational studies," PLoS ONE, vol. 9, no. 12, Article ID el16327, 2014.

[16] B. Viollet, B. Guigas, N. Sanz Garcia, J. Leclerc, M. Foretz, and F. Andreelli, "Cellular and molecular mechanisms of metformin: an overview," Clinical Science, vol. 122, no. 6, pp. 253-270, 2012.

[17] E. Giovannucci, D. M. Harlan, M. C. Archer et al., "Diabetes and cancer: a consensus report," Diabetes Care, vol. 33, no. 7, pp. 1674-1685, 2010.

[18] C.-H. Tseng, "Metformin and endometrial cancer risk in Chinese women with type 2 diabetes mellitus in Taiwan," Gynecologic Oncology, vol. 138, no. 1, pp. 147-153, 2015.

[19] M. Franchi, R. Asciutto, F. Nicotra et al., "Metformin, other antidiabetic drugs, and endometrial cancer risk: a nested casecontrol study within Italian healthcare utilization databases," European Journal of Cancer Prevention, 2016.

[20] E. M. Ko, P. Walter, A. Jackson et al., "Metformin is associated with improved survival in endometrial cancer," Gynecologic Oncology, vol. 132, no. 2, pp. 438-442, 2014.

[21] M. M. Al Hilli, J. N. Bakkum-Gamez, A. Mariani et al., "The effect of diabetes and metformin on clinical outcomes is negligible in risk-adjusted endometrial cancer cohorts," Gynecologic Oncology, vol. 140, no. 2, pp. 270-276, 2016.

[22] C. Becker, S. S. Jick, C. R. Meier, and M. Bodmer, "Metformin and the risk of endometrial cancer: a case-control analysis," Gynecologic Oncology, vol. 129, no. 3, pp. 565-569, 2013.

[23] J. Luo, S. Beresford, C. Chen et al., "Association between diabetes, diabetes treatment and risk of developing endometrial cancer," British Journal of Cancer, vol. 111, no. 7, pp. 1432-1439, 2014.

[24] E. M. Ko, T. Stürmer, J.-L. Hong, W. C. Castillo, V. Bae-Jump, and M. J. Funk, "Metformin and the risk of endometrial cancer: a population-based cohort study," Gynecologic Oncology, vol. 136, no. 2, pp. 341-347, 2015.

[25] N. S. Nevadunsky, A. Van Arsdale, H. D. Strickler et al., "Metformin use and endometrial cancer survival," Gynecologic Oncology, vol. 132, no. 1, pp. 236-240, 2014.

[26] A. Lemańska, M. Zaborowski, M. Spaczyński, and E. NowakMarkwitz, "Do endometrial cancer patients benefit from metformin intake?" Ginekologia Polska, vol. 86, no. 6, pp. 419-423, 2015.

[27] O. Ezewuiro, T. A. Grushko, M. Kocherginsky et al., "Association of metformin use with outcomes in advanced endometrial cancer treated with chemotherapy," PLoS ONE, vol. 11, no. 1, Article ID e0147145, 2016.

[28] V. Seebacher, B. Bergmeister, C. Grimm, H. Koelbl, A. Reinthaller, and S. Polterauer, "The prognostic role of metformin in patients with endometrial cancer: a retrospective 
study," European Journal of Obstetrics Gynecology and Reproductive Biology, vol. 203, pp. 291-296, 2016.

[29] A. Stang, "Critical evaluation of the Newcastle-Ottawa scale for the assessment of the quality of nonrandomized studies in metaanalyses," European Journal of Epidemiology, vol. 25, no. 9, pp. 603-605, 2010.

[30] K.-M. Lee, M. Lee, J. Lee et al., "Enhanced anti-tumor activity and cytotoxic effect on cancer stem cell population of metformin-butyrate compared with metformin $\mathrm{HCl}$ in breast cancer," Oncotarget, vol. 7, no. 25, pp. 38500-38512, 2016.

[31] Z. Al-Wahab, I. Mert, C. Tebbe et al., "Metformin prevents aggressive ovarian cancer growth driven by high-energy diet: similarity with calorie restriction," Oncotarget, vol. 6, no. 13, pp. 10908-10923, 2015.

[32] X. Xiao, Q. He, C. Lu et al., "Metformin impairs the growth of liver kinase B1-intact cervical cancer cells," Gynecologic Oncology, vol. 127, no. 1, pp. 249-255, 2012.

[33] A. Gadducci, N. Biglia, R. Tana, S. Cosio, and M. Gallo, "Metformin use and gynecological cancers: a novel treatment option emerging from drug repositioning," Critical Reviews in Oncology/Hematology, vol. 105, pp. 73-83, 2016.

[34] X. Yu, W. Mao, Y. Zhai et al., "Anti-tumor activity of metformin: from metabolic and epigenetic perspectives," Oncotarget, vol. 8, no. 3, pp. 5619-5628, 2016.

[35] S. A. Hawley, A. E. Gadalla, G. S. Olsen, and D. Grahame Hardie, "The antidiabetic drug metformin activates the AMP-activated protein kinase cascade via an adenine nucleotide-independent mechanism," Diabetes, vol. 51, no. 8, pp. 2420-2425, 2002.

[36] V. Brower, "Illuminating the diabetes-cancer link," Journal of the National Cancer Institute, vol. 104, no. 14, pp. 1048-1050, 2012.

[37] B. Jiang, X. Zhang, L.-L. Du et al., "Possible roles of insulin, IGF1 and IGFBPs in initiation and progression of colorectal cancer," World Journal of Gastroenterology, vol. 20, no. 6, pp. 1608-1613, 2014.

[38] K. M. J. De Bruijn, L. R. Arends, B. E. Hansen, S. Leeflang, R. Ruiter, and C. H. J. Van Eijck, "Systematic review and meta-analysis of the association between diabetes mellitus and incidence and mortality in breast and colorectal cancer," British Journal of Surgery, vol. 100, no. 11, pp. 1421-1429, 2013.

[39] E. S. Schernhammer, J. M. Holly, D. J. Hunter, M. N. Pollak, and S. E. Hankinson, "Insulin-like growth factor-I, its binding proteins (IGFBP-1 and IGFBP-3), and growth hormone and breast cancer risk in The Nurses Health Study II," EndocrineRelated Cancer, vol. 13, no. 2, pp. 583-592, 2006.

[40] R. N. Roy, A. H. Gerulath, A. Cecutti, and B. R. Bhavnani, "Discordant expression of insulin-like growth factors and their receptor messenger ribonucleic acids in endometrial carcinomas relative to normal endometrium," Molecular and Cellular Endocrinology, vol. 153, no. 1-2, pp. 19-27, 1999.

[41] E. Rozengurt, J. Sinnett-Smith, and K. Kisfalvi, "Crosstalk between insulin/insulin-like growth factor-1 receptors and G protein-coupled receptor signaling systems: a novel target for the antidiabetic drug metformin in pancreatic cancer," Clinical Cancer Research, vol. 16, no. 9, pp. 2505-2511, 2010.

[42] J. J. Howell, S. J. H. Ricoult, I. Ben-Sahra, and B. D. Manning, "A growing role for mTOR in promoting anabolic metabolism," Biochemical Society Transactions, vol. 41, no. 4, pp. 906-912, 2013.

[43] S. Sośnicki, M. Kapral, and L. Węglarz, "Molecular targets of metformin antitumor action," Pharmacological Reports, vol. 68, no. 5, pp. 918-925, 2016.
[44] Z.-H. Zhang, P.-Y. Su, J.-H. Hao, and Y.-H. Sun, "The role of preexisting diabetes mellitus on incidence and mortality of endometrial cancer: a meta-analysis of prospective cohort studies," International Journal of Gynecological Cancer, vol. 23, no. 2, pp. 294-303, 2013.

[45] J. Xie, X. Wang, and C. G. Proud, "mTOR inhibitors in cancer therapy," F1000Research, vol. 5, 2016. 


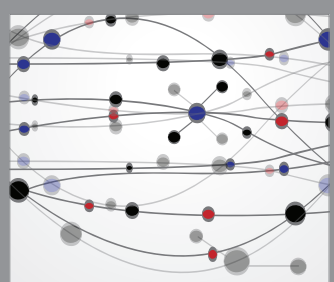

The Scientific World Journal
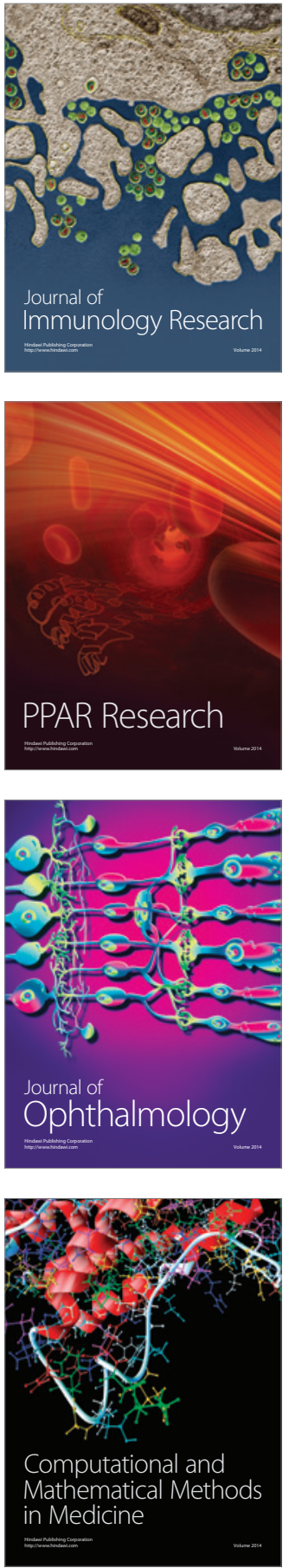

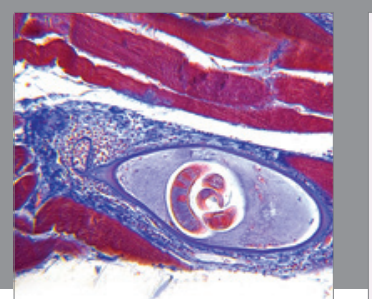

Gastroenterology Research and Practice
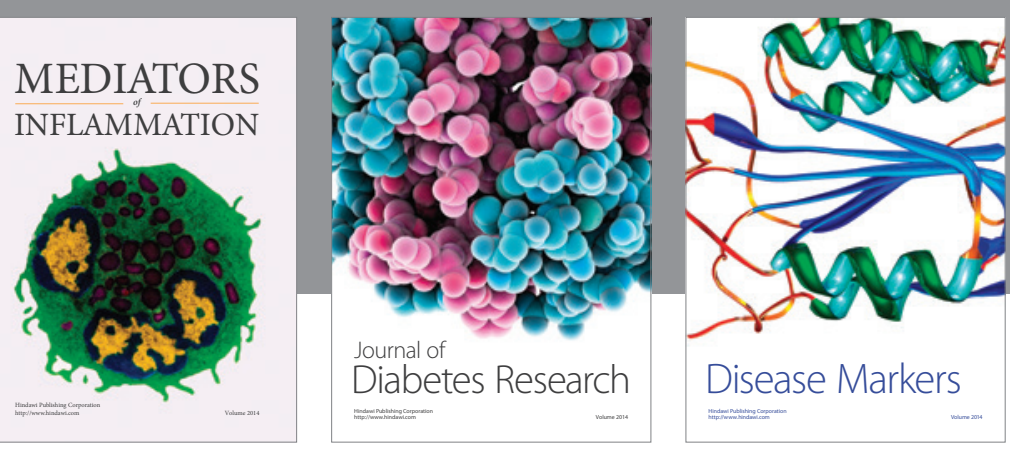

Disease Markers

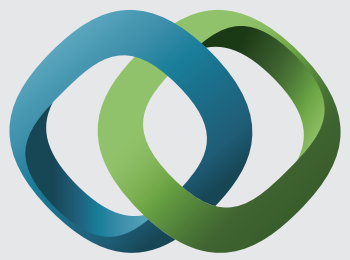

\section{Hindawi}

Submit your manuscripts at

https://www.hindawi.com
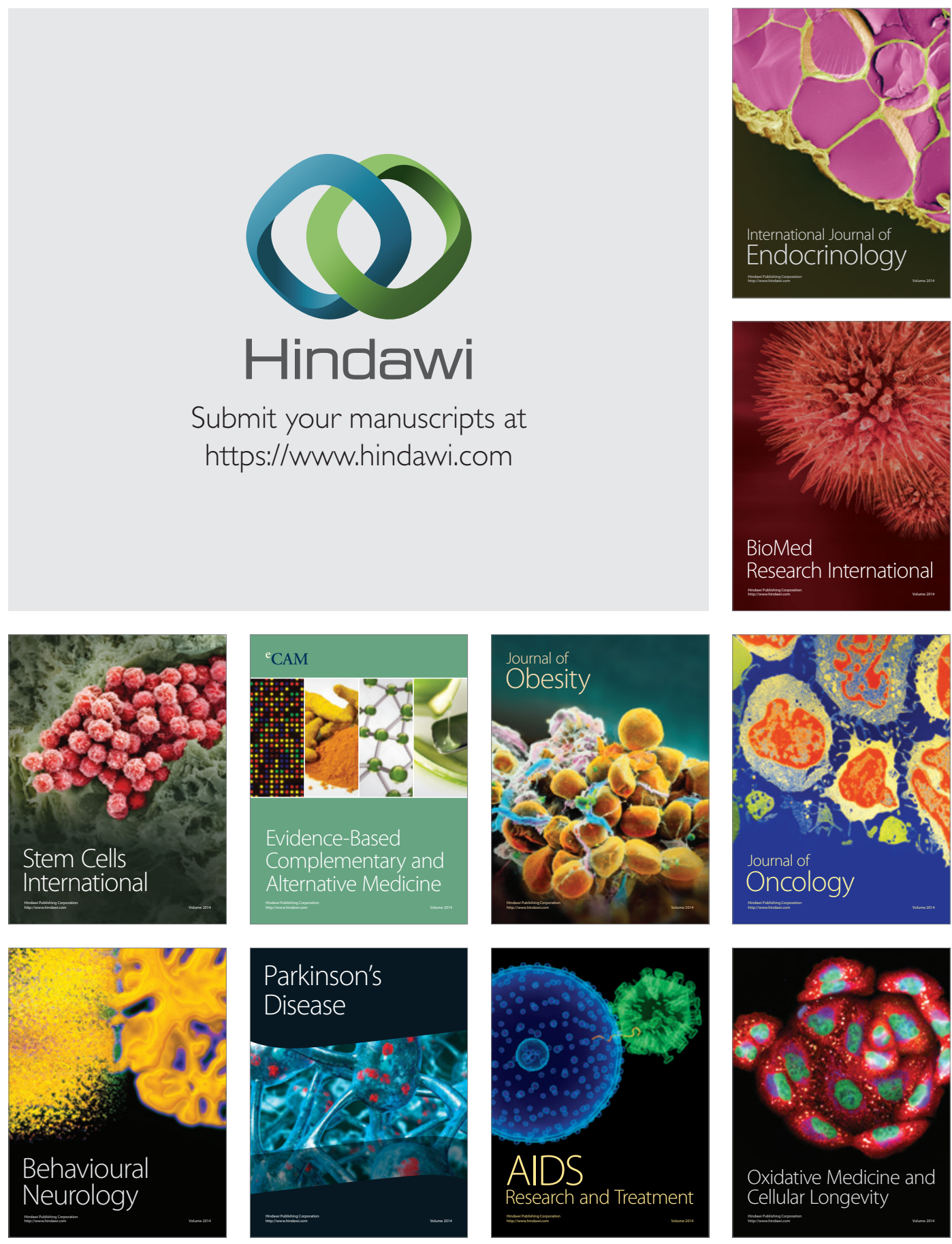